\title{
Intrinsic Josephson junctions in the iron-based multi-band superconductor $\left(\mathrm{V}_{2} \mathrm{Sr}_{4} \mathrm{O}_{6}\right) \mathrm{Fe}_{2} \mathrm{As}_{2}$
}

\author{
Philip J. W. Moll ${ }^{1 \star}$, Xiyu Zhu ${ }^{2}$, Peng Cheng ${ }^{3 \dagger}$, Hai-Hu Wen ${ }^{2,3}$ and Bertram Batlogg
}

In layered superconductors, Josephson junctions may be formed within the unit celli-3 as a result of sufficiently low inter-layer coupling. These intrinsic Josephson junction (iJ) systems ${ }^{4}$ have attracted considerable interest for their application potential in quantum computing as well as efficient sources of $\mathrm{THz}$ radiation, closing the famous ' $\mathrm{THz} \mathrm{gap}^{15}$. So far, iJJ have been demonstrated in single-band, copper-based high- $\mathrm{T}_{\mathrm{c}}$ superconductors, mainly in Bi-Sr-Ca-Cu-O (refs 6-8). Here we report clear experimental evidence for $\mathrm{i} J \mathrm{~J}$ behaviour in the iron-based superconductor $\left(\mathrm{V}_{2} \mathrm{Sr}_{4} \mathrm{O}_{6}\right) \mathrm{Fe}_{2} \mathrm{As}_{2}$. The intrinsic junctions are identified by periodic oscillations of the flux-flow voltage on increasing a well-aligned in-plane magnetic field ${ }^{9}$. The periodicity is explained by commensurability effects between the Josephson vortex lattice and the crystal structure, which is a hallmark signature of Josephson vortices confined into iJJ stacks ${ }^{10,11}$. This finding adds the pnictide $\left(\mathrm{V}_{2} \mathrm{Sr}_{4} \mathrm{O}_{6}\right) \mathrm{Fe}_{2} \mathrm{As}_{2}$ to the copper-based $\mathrm{iJJ}$ materials of interest for Josephson junction applications. In particular, novel devices based on multi-band Josephson coupling may be realized.

Applications based on Josephson junctions, such as superconducting quantum interference device (SQUID) magnetometers, exploit the phase coherence of the macroscopic wavefunction of a superconductor. In these successful applications, artificially grown heterostructures incorporating a layer of order parameter suppression-typically an oxide barrier-serve as the Josephson junctions. With the discovery of cuprate high- $T_{c}$ superconductors and their small out-of-plane coherence length resulting from insulating layers separating the $\mathrm{Cu}-\mathrm{O}$ planes, the idea of intrinsic Josephson junctions (iJJ) inside the crystal structure and, consequently, devices based on self-assembled nano-junctions quickly emerged.

With the advent of iron-based superconductors, the iron pnictides, as a different class of layered high- $T_{\mathrm{c}}$ superconductors, the question of their potential as iJJ systems arises naturally. Of particular interest for application is the physical realization of an iJJ system with multi-band superconductivity, which is expected to influence the Josephson junction behaviour. Novel effects and applications have been proposed that do not occur in single-band superconductors such as cuprates: Josephson vortices in intrinsic $s_{ \pm}$junctions are predicted to show a significantly extended core region compared with single-gap materials owing to destructive interference of the Cooper pair tunnelling channels ${ }^{12}$, which may lead to a difference in interaction with pinning centres. The differences in phase evolution around the phase-core region of a Josephson vortex, which arise from the extra inter- and intra-band tunnelling channels, may also be exploited in phase sensitive iJJ applications such as quantum computing ${ }^{7,13}$.

One application example is the efficient generation of terahertz radiation due to the ac-Josephson effect, aimed at closing the famous 'THz gap': in between the realms of solid state emitters at lower frequencies and optical emitters at higher frequencies, a frequency range around a few $\mathrm{THz}$ lacks efficient, compact and cost-effective emitters and detectors. Until now all such emitters have been based on cuprates, mainly on the highly anisotropic $\mathrm{Bi}-\mathrm{Sr}-\mathrm{Ca}-\mathrm{Co}-\mathrm{O}$ (BSCCO). The extra inter-band channel in multiband $\mathrm{iJJ}$ is expected to lead to a new type of inter-layer coupling, related to the Leggett mode $\mathrm{e}^{14}$. It has been shown theoretically to favour a $\pi$-phase difference between adjacent layers and thus aids in the junction synchronization through an iJJ $\operatorname{stack}^{15,16}$, as well as in altering the $I-V$ characteristics ${ }^{17}$. Such junction phase synchronization is essential to achieve high-power $\mathrm{THz}$ radiation output, which in single-band materials has been achieved through a cavity resonance condition ${ }^{18}$. The high levels of output power of such resonator stacks, however, come at the price of lost frequency tunability. If, indeed, the inter-band tunnelling channel aids interlayer synchronization, a new path to tunable, high-power $\mathrm{THz}$ sources will emerge.

Continuing theoretical studies of Josephson effects in multiband systems have indicated several iron pnictides as candidates for iJJ behaviour, as well as potential experimental signatures of such effects having been reported ${ }^{19,20}$. Such layered compounds, however, are commonly known to incorporate inter-growth layers, which may thus naturally form extrinsic Josephson junctions within the 'single crystal'. It is an experimental challenge to carefully distinguish such extrinsic contributions from true iJJ behaviour originating from order parameter modulations within the unit cell. The key aspect of this study is to provide clear and unambiguous evidence for iJJ behaviour in an iron pnictide superconductor and thus experimentally show the existence of intrinsic multi-band Josephson junctions on an atomic scale.

For intrinsic Josephson coupling, the $c$-axis coherence length $\xi_{c}$ must be smaller than the inter-layer spacing ${ }^{21}$. The least anisotropic pnictide family that falls under this category is the ' 1111 ' class, including the highest $T_{c}$ pnictide $\operatorname{SmFeAs}(\mathrm{O}, \mathrm{F})$. This system has been reported to be on the verge of Josephson behaviour, showing Josephson-like in-plane vortices ${ }^{20,22}$. The low $c$-axis resistivity as well as the high critical current density perpendicular to the planes ${ }^{23}$, however, indicate a very small inter-layer barrier height, impeding true iJJ behaviour and thus limiting this material's prospect for iJJ applications.

\footnotetext{
${ }^{1}$ Physics of New Materials, Solid State Physics, ETH Zürich, CH-8093 Zürich, Switzerland, ${ }^{2}$ Center for Superconducting Physics and Materials, National Laboratory of Solid State Microstructures and Department of Physics, Nanjing University, Nanjing 210093, China, ${ }^{3}$ National Laboratory for Superconductivity, Institute of Physics, Chinese Academy of Sciences, Beijing 100190, China. 'Present address: Department of Physics, Renmin University of China, Beijing 100872, China. *e-mail: phmoll@phys.ethz.ch
} 

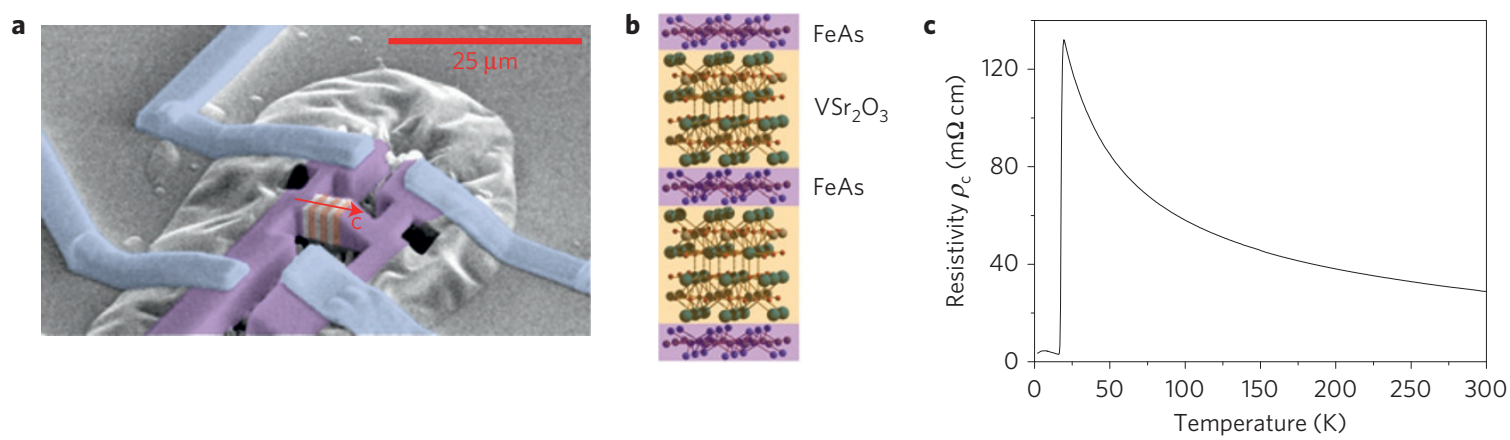

Figure 1 | The intrinsic Josephson junction pnictide $\left(\mathrm{V}_{2} \mathrm{Sr}_{4} \mathrm{O}_{6}\right) \mathrm{Fe}_{2} \mathrm{As}_{2}$. $\mathbf{a}$, Scanning electron micrograph of the microstructured crystal of $\left(\mathrm{V}_{2} \mathrm{Sr}_{4} \mathrm{O}_{6}\right) \mathrm{Fe}_{2} \mathrm{As}_{2}$ (purple). The c-direction resistivity is measured in a four-point geometry that was carved out of a single crystal by focused ion beam (FIB) cutting and contacted via FIB-deposited Pt-leads (blue). The active Josephson junction stack (red and white planes) is between the voltage contacts, and the direction of the layers is indicated (red arrow). $\mathbf{b}$, Crystal structure of $\left(\mathrm{V}_{2} \mathrm{Sr}_{4} \mathrm{O}_{6}\right) \mathrm{Fe}_{2} \mathrm{As}_{2}$, depicting the intrinsic tunnel barrier $\mathrm{VSr}_{2} \mathrm{O}_{3}$-blocks between the superconducting FeAs layers. c, c-direction resistivity of $\left(\mathrm{V}_{2} \mathrm{Sr}_{4} \mathrm{O}_{6}\right) \mathrm{Fe}_{2} \mathrm{As}_{2}$ as a function of temperature. On cooling, the resistivity increases, indicating the insulating nature of the $\mathrm{VSr}_{2} \mathrm{O}_{3}$-blocks. The transition temperature $T_{\mathrm{c}}$ is $19 \mathrm{~K}$, indicating slightly underdoped samples $\left(\mathrm{V}_{2} \mathrm{Sr}_{4} \mathrm{O}_{5.2}\right) \mathrm{Fe}_{2} \mathrm{As}_{2}$ (ref. 30). The non-vanishing resistance below $T_{\mathrm{c}}$ indicates the presence of inter-growth defects. The weak temperature dependence below $T_{\mathrm{C}}$ suggests the defect contribution to the total resistivity to be small.

Therefore we focus in this study on a more decoupled pnictide, $\left(\mathrm{V}_{2} \mathrm{Sr}_{4} \mathrm{O}_{6}\right) \mathrm{Fe}_{2} \mathrm{As}_{2}$, a member of the '24622' structure class with a large inter-layer spacing of $d_{\mathrm{c}}=1.56 \mathrm{~nm}$ (ref. 24). This compound is a promising iJJ candidate among the long- $c$-axis pnictides, as the $\left(\mathrm{VSr}_{2} \mathrm{O}_{3}\right)$ layers have been predicted to be insulating due to $\mathrm{V}-d$ orbital correlations from calculations using the local density approximation with the Hubbard correction $(\mathrm{LDA}+\mathrm{U})$ (ref. 25). These correlations have been confirmed by photoemission ${ }^{26}$ to lead to a Mott insulator separating the superconducting FeAs layers. The influence of the material's layered structure on the vortex matter and on its behaviour as an intrinsic Josephson system can be best studied by electric transport measurements perpendicular to the layers-that is, along the $c$ direction. Owing to the small size and unfavourable plate-like shape of available single crystals, the samples were microfabricated and contacted using the focused ion beam (FIB) technique ${ }^{23}$ : in this process, an individual crystallite was selected and carved into its final form, shown in Fig. 1a, by a $\mathrm{Ga}^{2+}$ ion beam: the crystal (purple) has been carved into a fourpoint geometry, and was contacted by FIB-platinum deposition (blue). This technique allows reliable measurements of the out-ofplane resistivity $\rho_{\mathrm{c}}$, which is otherwise inaccessible to transport experiments in microcrystallites.

Figure 1c shows the temperature dependence of the $c$-direction resistivity $\rho_{c}(T)$. The resistivity increases from $29 \mathrm{~m} \Omega \mathrm{cm}$ at room temperature to $131 \mathrm{~m} \Omega \mathrm{cm}$ at $T_{\mathrm{c}} \sim 19.8 \mathrm{~K}$, following a power law dependence $\rho_{\mathrm{c}}(T) \sim T^{-0.5}$. Such a temperature dependence of the out-of-plane resistivity is experimental evidence for incoherent electron transport, as expected for the Mott-insulating $\mathrm{VSr}_{2} \mathrm{O}_{3}$ layers. Therefore, superconductor-insulator-superconductor (SIS) junctions are expected instead of superconductor-normal metalsuperconductor (SNS)-type junctions.

This overall temperature dependence of $\rho_{\mathrm{c}}$ is qualitatively reminiscent of the more anisotropic cuprates; however, the absolute value of $\rho_{\mathrm{c}}\left(T_{\mathrm{c}}\right)$ is two orders of magnitude smaller than the highly anisotropic iJJ compounds $\mathrm{Bi}_{2} \mathrm{Sr}_{2} \mathrm{CaCu}_{2} \mathrm{O}_{8+x}(10-30 \Omega \mathrm{cm})$ and $\mathrm{Tl}_{2} \mathrm{Ba}_{2} \mathrm{Ca}_{2} \mathrm{Cu}_{3} \mathrm{O}_{10}(13 \Omega \mathrm{cm}$; ref. 3), but higher than the more isotropic pnictide $\mathrm{SmFeAs}(\mathrm{O}, \mathrm{F})(2.7 \mathrm{~m} \Omega \mathrm{cm}$; ref. 23). This lower out-of-plane resistivity in $\left(\mathrm{V}_{2} \mathrm{Sr}_{4} \mathrm{O}_{6}\right) \mathrm{Fe}_{2} \mathrm{As}_{2}$ is indicative of a stronger inter-layer coupling than in $\mathrm{Bi}_{2} \mathrm{Sr}_{2} \mathrm{CaCu}_{2} \mathrm{O}_{8+x}$.

The key observation revealing the intrinsic Josephson effect is the vortex matter behaviour when the magnetic field is well aligned parallel to the planes and a d.c.-current flows perpendicular to the layers (Fig. 2). In this configuration, the Lorentz force induces an in-plane sliding motion of vortices parallel to the superconducting layers, as sketched in Fig. 2a. Measurable flux flow starts just above $1 \mathrm{~T}$ and increases monotonically with increasing field. In fields between $2.5 \mathrm{~T}$ and $6 \mathrm{~T}$, well-defined periodic oscillations appear on an increasing background. The oscillation period is constant over the whole field range, with a periodicity $H_{\mathrm{P}} \approx 0.178 \mathrm{~T}$, and the oscillations persist over an extended temperature window from $15 \mathrm{~K}$ down to $2 \mathrm{~K}$, the lowest accessible temperature in the experiments.

This periodic modulation of the out-of-plane flux-flow voltage on increasing a well-aligned in-plane field is a hallmark of intrinsic Josephson behaviour. Such oscillatory effects have been extensively studied in cuprate iJJ compounds ${ }^{6,9,27,28}$ and their origin has been theoretically explained as a result of a periodic modulation of the surface barrier due to vortex matching effects ${ }^{10,11}$. For magnetic fields well aligned with the superconducting layers, vortices in an iJJ system are purely of Josephson character, without any Abrikosovlike segments ('pancake vortices'). In this case, vortex motion perpendicular to the layers-that is, across the superconducting barrier-is suppressed and this intrinsic vortex barrier is sometimes referred to as intrinsic pinning ${ }^{2}$. The Josephson vortices, however, are much more weakly pinned by defects owing to the absence of a normal $\operatorname{cor}^{21}$, and thus are highly mobile between two adjacent FeAs planes ${ }^{22}$. Without effective defect pinning, the main source of pinning is the surface barrier that impedes vortex entry and exit. This surface barrier is known to be modulated by commensurability effects between the layered crystal structure and the Josephson vortex lattice, and thus gives rise to the observed oscillations.

The periodicity $H_{\mathrm{P}}$ corresponds to the field required to add one flux quantum into every second $\mathrm{VSr}_{2} \mathrm{O}_{3}$ layer forming a hexagonal arrangement of vortices. The vortex structure associated with these commensurability oscillations is sketched in Fig. 2a for two exemplary cases. These two configurations differ by one extra vortex in every second layer, and the transition between them requires a change by one flux quantum $\Phi_{0}$ per area $\left(2 d_{c}\right) \times w$, where $w$ denotes the sample width. Therefore the oscillation period depends on the material specific inter-layer distance $d_{c}$ as well as on the sample geometry, as has been shown in cuprates ${ }^{9}$ :

$$
H_{\mathrm{P}}=\frac{\Phi_{0}}{2 w d_{\mathrm{c}}}
$$

Using the $c$-axis lattice parameter determined by X-ray diffraction as $d_{c}=1.567 \mathrm{~nm}$ (ref. 24) and the width of the FIB-cut sample measured by scanning electron microscopy as $w=3.6 \mu \mathrm{m}$, the calculated periodicity field $H_{\mathrm{P}}=0.177 \mathrm{~T}$ is in perfect agreement with 


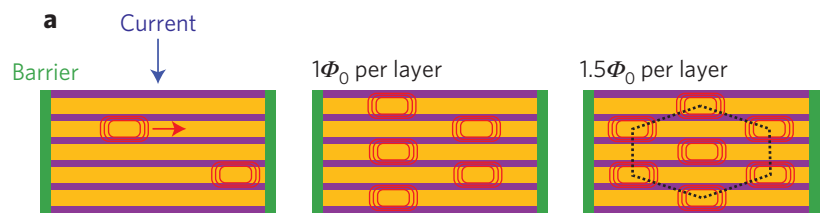

b
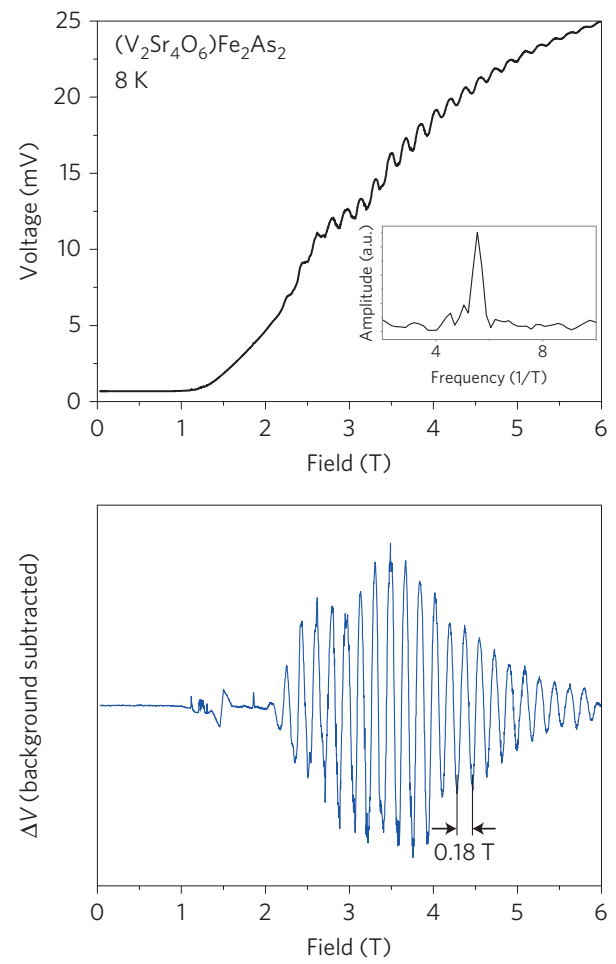

Figure $\mathbf{2}$ | Josephson vortex commensurability oscillations. a, Sketch of the vortex motion in the iJJ stack. The out-of-plane current drives the Josephson vortices in a sliding motion through the structure. Although point pinning is ineffective for Josephson vortices, the surface barrier impedes vortex entry or exit. The middle and right panels show a sketch of two neighbouring commensurate vortex configurations. On increasing the field from the middle configuration at $1 \Phi_{0}$ per layer, the next commensurate configuration is reached at $1.5 \Phi_{0}$ per layer. Therefore a periodicity of one flux quantum per two unit layers corresponds to the observed vortex lattice periodicity. The dotted line indicates the hexagonal unit cell of the observed vortex lattice. b, Top: flux-flow voltage at a constant d.c.-current of $20 \mu \mathrm{A}$ as a function of in-plane magnetic field. Inset: frequency spectrum of the signal. Bottom: as above, but with background subtracted. At high fields above $2.5 \mathrm{~T}$, pronounced oscillations appear on the otherwise monotonically increasing background of flux motion. These oscillations are periodic in field, and only one significant periodicity of $0.178 \mathrm{~T}$ is observed.

the observed periodicity of $0.178 \mathrm{~T}$. The oscillations are expected to occur only at high vortex densities, in which the Josephson junctions are homogeneously penetrated by vortices. This penetration field has been theoretically predicted ${ }^{10}$ as $H_{\mathrm{cr}}=\Phi_{0} / 2 \pi \gamma d_{\mathrm{c}}^{2}$, where $\gamma$ denotes the coherence length anisotropy. As the onset of oscillations is observed at about $2.5 \mathrm{~T}$, the anisotropy can thus be estimated as $\gamma \approx 51$. This reasonable value is significantly lower than typical values of $\gamma>1,000$ in $\mathrm{Bi}_{2} \mathrm{Sr}_{2} \mathrm{CaCu}_{2} \mathrm{O}_{8+x}$, in line with the smaller outof-plane resistance in $\left(\mathrm{V}_{2} \mathrm{Sr}_{4} \mathrm{O}_{6}\right) \mathrm{Fe}_{2} \mathrm{As}_{2}$ (ref. 3).

An important aspect of the Josephson oscillations is their robustness against material defects, clearly excluding extrinsic Josephson junctions, such as planar inter-growth layers, as the origin of these observations. In all studied samples, a small nonzero resistance was observed below $T_{c}$, most likely as a result of layered defects. Such layered defects are common in long-c-axis

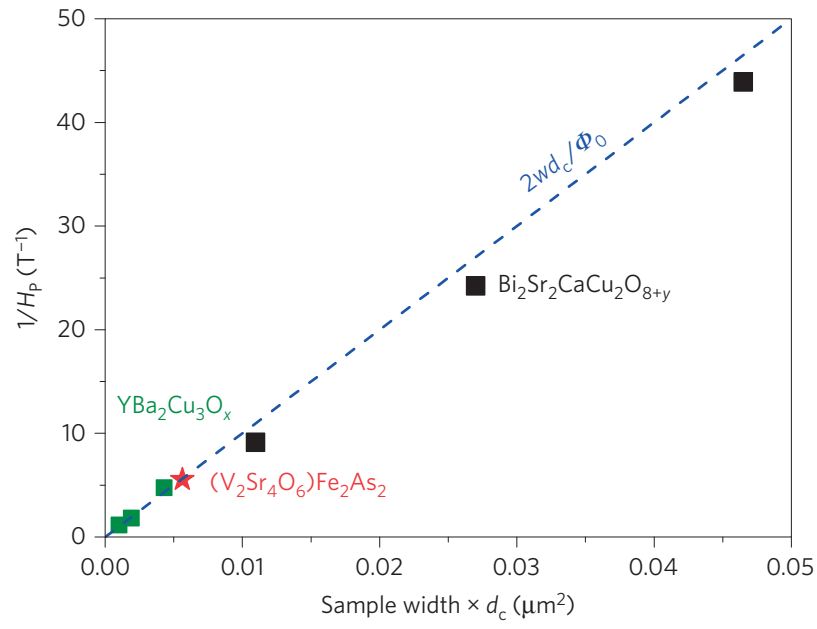

Figure 3 | Comparison against copper-based intrinsic Josephson junction compounds. Comparison of the field periodicity of the intrinsic Josephson oscillations from the iron pnictide $\left(\mathrm{V}_{2} \mathrm{Sr}_{4} \mathrm{O}_{6}\right) \mathrm{Fe}_{2} \mathrm{As}_{2}$ (this work; red) against the cuprates $\mathrm{Bi}_{2} \mathrm{Sr}_{2} \mathrm{CaCu}_{2} \mathrm{O}_{8+y}$ (black; ref. 9) and $\mathrm{YBa}_{2} \mathrm{Cu}_{3} \mathrm{O}_{x}$ (green; ref. 28). These compounds all show Josephson oscillation periods in good agreement with the theoretically expected $1 / H_{p}=2 w d_{c} / \Phi_{0}$. This provides evidence that similar inter-layer Josephson vortex arrangements are realized in $\left(\mathrm{V}_{2} \mathrm{Sr}_{4} \mathrm{O}_{6}\right) \mathrm{Fe}_{2} \mathrm{As}_{2}$ and cuprate superconductors, despite their distinct physics.

materials, and highlight the complex chemistry and synthesis challenges in this quinary oxide. Owing to this finite resistance in the superconducting state, self-heating prevented the observation of branches in the $I-V$ characteristics, another key evidence for intrinsic Josephson junctions ${ }^{29}$. These defect layers, however, do not contribute to the oscillatory signal unless they occur in periodic arrays along the $c$ direction and are all of the exact same thickness. The absence of further frequencies, as shown in the inset of Fig. 2, and the coincidence of the observed effective Josephson junction thickness with the material parameter $d_{c}$ clearly exclude this highly unlikely scenario.

The similarity of the oscillatory enhancement of flux-flow dissipation between $\left(\mathrm{V}_{2} \mathrm{Sr}_{4} \mathrm{O}_{6}\right) \mathrm{Fe}_{2} \mathrm{As}_{2}$ and $\mathrm{Bi}_{2} \mathrm{Sr}_{2} \mathrm{CaCu}_{2} \mathrm{O}_{8+x}$ is evident from Fig. 3. The experimentally observed oscillation periods in both compound families closely follow the linear relation given by equation (1). In particular, as not only fundamental constants but also the sample geometry determine the oscillation period $H_{\mathrm{P}}$ (equation (1)), the collapse of the very different oscillation periods measured in samples of different shape in different materials onto a universal curve indicates the common intrinsic Josephson physics.

It will be interesting to further explore the new multi-band twist in iJJ physics that has now been shown to be experimentally accessible in iron pnictides. We hope these findings will stimulate further experimental and theoretical efforts to investigate the interplay between the intra- and inter-band tunnelling channels, uncovering their role in new quantum coherent processes in the junction and ultimately leading to their exploitation in technology.

\section{Methods}

Crystal growth. The high-quality single crystals of $\mathrm{V}_{2} \mathrm{Sr}_{4} \mathrm{O}_{6} \mathrm{Fe}_{2} \mathrm{As}_{2}$ were grown by the flux method. First the starting materials $-\mathrm{V}_{2} \mathrm{O}_{5}$ powder (purity $99.95 \%$ ), $\mathrm{Fe}_{2} \mathrm{O}_{3}$ powder (purity 99.9\%), Sr grains (purity 99\%) and $\mathrm{FeAs}$ powder-were mixed in stoichiometry according to $\mathrm{V}_{2} \mathrm{Sr}_{4} \mathrm{O}_{6} \mathrm{Fe}_{2} \mathrm{As}_{2}$. Then the powder was ground with more FeAs powder of molar ratio ( $\mathrm{FeAs}: \mathrm{V}_{2} \mathrm{Sr}_{4} \mathrm{O}_{6} \mathrm{Fe}_{2} \mathrm{As}_{2}=2: 1$ ), sealed in an evacuated quartz tube and heated at about $1,150^{\circ} \mathrm{C}$ for two days. Then it was cooled at a rate of $3{ }^{\circ} \mathrm{Ch}^{-1}$ to $1,050^{\circ} \mathrm{C}$, followed by a rapid cooling down by shutting off the power of the furnace. The resulting product is a pellet with a dark colour that contains mainly plate-like small crystals. 
Measurements. Resistivity measurements were performed in quasi-d.c. mode by applying rectangular current pulses. For all measurements, a Keithley Nanovolt option was used in the 'Delta Mode' setting, thus switching the current from $+I$ to $-I$ every $83 \mathrm{~ms}$. The field was well aligned with the FeAs layers using an Attocube slip-stick rotator by maximizing the flux-flow motion in a constant applied field.

Received 2 October 2013; accepted 16 June 2014; published online 27 July 2014

\section{References}

1. Tachiki, M. \& Takahashi, S. Strong vortex pinning intrinsic in high- $T_{\mathrm{c}}$ oxide superconductors. Solid State Commun. 70, 291-295 (1989).

2. Feinberg, D. \& Villard, C. Intrinsic pinning and lock-in transition of flux lines in layered type-II superconductors. Phys. Rev. Lett. 65, 919-922 (1990).

3. Kleiner, R. \& Müller, P. Intrinsic Josephson effects in high- $T_{\mathrm{c}}$ superconductors. Phys. Rev. B 49, 1327-1343 (1994)

4. Kwok, W. K. et al. Direct observation of intrinsic pinning by layered structure in single-crystal $\mathrm{YBa}_{2} \mathrm{Cu}_{3} \mathrm{O}_{7-\delta}$. Phys. Rev. B 67, 390-393 (1991).

5. Tonouchi, M. Cutting-edge terahertz technology. Nature Photon. 1, 97-105 (2007).

6. Vedeneev, S. \& Maude, D. In-plane current-voltage characteristics and oscillatory Josephson-vortex flow resistance in $\mathrm{La}$-free $\mathrm{Bi}_{2+x} \mathrm{Sr}_{2-x} \mathrm{CuO}_{6+\delta}$ single crystals in high magnetic fields. Phys. Rev. B 77, 064511 (2008).

7. Li, S-X. et al. Observation of macroscopic quantum tunneling in a single $\mathrm{Bi}_{2} \mathrm{Sr}_{2} \mathrm{CaCu}_{2} \mathrm{O}_{8+\delta}$ surface intrinsic Josephson junction. Phys. Rev. Lett. 99, 037002 (2007).

8. Schlenga, K. et al. Subgap structures in intrinsic Josephson junctions of $\mathrm{Tl}_{2} \mathrm{Ba}_{2} \mathrm{Ca}_{2} \mathrm{Cu}_{3} \mathrm{O}_{10+d}$ and $\mathrm{Bi}_{2} \mathrm{Sr}_{2} \mathrm{CaCu}_{2} \mathrm{O}_{8+d}$. Phys. Rev. Lett. 76, 4943-4946 (1996).

9. Ooi, S., Mochiku, T. \& Hirata, K. Periodic oscillations of Josephson-vortex flow resistance in $\mathrm{Bi}_{2} \mathrm{Sr}_{2} \mathrm{CaCu}_{2} \mathrm{O}_{8+y}$. Phys. Rev. Lett. 89, 247002 (2002).

10. Koshelev, A. Magnetic oscillations of critical current in intrinsic Josephson-junction stacks. Phys. Rev. B 75, 214513 (2007).

11. Machida, M. Dynamical matching of Josephson vortex lattice with sample edge in layered high- $T_{\mathrm{c}}$ superconductors: Origin of the periodic oscillation of flux flow resistance. Phys. Rev. Lett. 90, 037001 (2003).

12. Ota, Y., Machida, M., Koyama, T. \& Matsumoto, H. Theory of vortex structure in Josephson junctions with multiple tunneling channels: Vortex enlargement as a probe of $\pm s$-wave superconductivity. Phys. Rev. B 81, 014502 (2010).

13. Inomata, K. et al. Macroscopic quantum tunneling in a d-wave high- $T_{c}$ $\mathrm{Bi}_{2} \mathrm{Sr}_{2} \mathrm{CaCu}_{2} \mathrm{O}_{8+\delta}$ superconductor. Phys. Rev. Lett. 95, 107005 (2005).

14. Leggett, A. J. Number-phase fluctuations in two-band superconductors. Prog. Theor. Phys. 36, 901-930 (1966).

15. Nakamura, H., Machida, M., Koyama, T. \& Hamada, N. First-principles study for the anisotropy of iron-based superconductors toward power and device applications. J. Phys. Soc. Jpn 78, 123712 (2009).

16. Ota, Y., Machida, M. \& Koyama, T. Variety of $c$-axis collective excitations in layered multigap superconductors. Phys. Rev. Lett. 106, 157001 (2011).

17. Koyama, T., Ota, Y. \& Machida, M. $I-V$ characteristics in multi-gap intrinsic Josephson junction stacks. Phys. C Supercond. 470, 1481-1484 (2010).
18. Ozyuzer, L. et al. Emission of coherent $\mathrm{THz}$ radiation from superconductors. Science 318, 1291-1293 (2007).

19. Kashiwaya, H. et al. $\mathrm{C}$-axis critical current of a $\mathrm{PrFeAsO}_{0.7}$ single crystal. Appl. Phys. Lett. 96, 202504 (2010).

20. Iida, K. et al. Oxypnictide $\mathrm{SmFeAs}(\mathrm{O}, \mathrm{F})$ superconductor: A candidate for high-field magnet applications. Sci. Rep. 3, 2139 (2013).

21. Blatter, G., Feigel'man, M., Geshkenbein, V., Larkin, A. \& Vinokur, V. Vortices in high-temperature superconductors. Rev. Mod. Phys. 66, 1125-1387 (1994).

22. Moll, P. J. W. et al. Transition from slow Abrikosov to fast moving Josephson vortices in iron pnictide superconductors. Nature Mater. 12, 134-138 (2013).

23. Moll, P. J. W. et al. High magnetic-field scales and critical currents in $\mathrm{SmFeAs}(\mathrm{O}, \mathrm{F})$ crystals. Nature Mater. 9, 628-633 (2010).

24. Zhu, X. et al. Transition of stoichiometric $\mathrm{Sr}_{2} \mathrm{VO}_{3} \mathrm{FeAs}$ to a superconducting state at 37.2 K. Phys. Rev. B 79, 220512 (2009)

25. Nakamura, H. \& Machida, M. Magnetic ordering in blocking layer and highly anisotropic electronic structure of high- $T_{\mathrm{c}}$ iron-based superconductor $\mathrm{Sr}_{2} \mathrm{VFeAsO}_{3}$ : LDA+U study. Phys. Rev. B 82, 094503 (2010).

26. Qian, T. et al. Quasinested Fe orbitals versus Mott-insulating V orbitals in superconducting $\mathrm{Sr}_{2} \mathrm{VFeAsO}_{3}$ as seen from angle-resolved photoemission. Phys. Rev. B 83, 140513 (2011)

27. Kadowaki, K. et al. Dynamical properties of Josephson vortices in mesoscopic intrinsic Josephson junctions in single crystalline $\mathrm{Bi}_{2} \mathrm{Sr}_{2} \mathrm{CaCu}_{2} \mathrm{O}_{8+\delta}$. Phys. C Supercond. 4370-438, 111-117 (2006).

28. Nagao, M. et al. Periodic oscillations of Josephson-vortex flow resistance in oxygen-deficient $\mathrm{YBa}_{2} \mathrm{Cu}_{3} \mathrm{O}_{x}$. Phys. Rev. B 74, 054502 (2006).

29. Rapp, M., Murk, A., Semerad, R. \& Prusseit, W. $c$-axis conductivity and intrinsic Josephson effects in $\mathrm{YBa}_{2} \mathrm{Cu}_{3} \mathrm{O}_{7-\delta}$. Phys. Rev. Lett. 77, 928-931 (1996).

30. Han, F. et al. Structural and transport properties of $\mathrm{Sr}_{2} \mathrm{VO}_{3-\delta} \mathrm{FeAs}$ superconductors with different oxygen deficiencies. Sci. China A $\mathbf{5 3}$, 1202-1206 (2010)

\section{Acknowledgements}

We thank G. Blatter and D. Geshkenbein for stimulating discussions. FIB work was supported by EMEZ and ScopeM at ETH Zürich. Work in Nanjing was supported by the Ministry of Science and Technology of China (973 Projects: No. 2011CBA00102, No. 2010СB923002).

\section{Author contributions}

P.J.W.M. designed the experiment, FIB-structured the crystals and performed the measurements. Single crystals were grown and analysed by X.Z., P.C. and H-H.W. P.J.W.M. and B.B. analysed the data and wrote the manuscript.

\section{Additional information}

Supplementary information is available in the online version of the paper. Reprints and permissions information is available online at www.nature.com/reprints. Correspondence and requests for materials should be addressed to P.J.W.M.

\section{Competing financial interests}

The authors declare no competing financial interests. 